\title{
Ensemble Empirical Mode Decomposition: Image Data Analysis with White-noise Reflection
}

\author{
M. Kopecký
}

\begin{abstract}
During the last decade, Zhaohua Wu and Norden E. Huang announced a new improvement of the original Empirical Mode Decomposition method (EMD). Ensemble Empirical Mode Decomposition and its abbreviation EEMD represents a major improvement with great versatility and robustness in noisy data filtering. EEMD consists of sifting and making an ensemble of a white noise-added signal, and treats the mean value as the final true result. This is due to the use of a finite, not infinitesimal, amplitude of white noise which forces the ensemble to exhaust all possible solutions in the sifting process. These steps collate signals of different scale in a proper intrinsic mode function (IMF) dictated by the dyadic filter bank. As EEMD is a time-space analysis method, the added white noise is averaged out with a sufficient number of trials. Here, the only persistent part that survives the averaging process is the signal component (original data), which is then treated as the true and more physically meaningful answer. The main purpose of adding white noise was to provide a uniform reference frame in the time-frequency space. The added noise collates the portion of the signal of comparable scale in a single IMF. Image data taken as time series is a non-stationary and nonlinear process to which the new proposed EEMD method can be fitted out. This paper reviews the new approach of using EEMD and demonstrates its use on the example of image data analysis, making use of some advantages of the statistical characteristics of white noise. This approach helps to deal with omnipresent noise.
\end{abstract}

Keywords: Empirical Mode Decomposition (EMD), Ensemble Empirical Mode Decomposition (EEMD), image analysis, sifting, Noise-Assigned Data Analysis (NADA).

\section{Introduction}

Empirical Mode Decomposition (EMD) has been proposed as an adaptive time-frequency data analysis method. It has been proved in applications for extracting signals from data generated in noisy nonlinear and non-stationary processes $[1,2]$. However, there are still several known unresolved difficulties with EMD. The first major weakness of the original EMD is the frequent occurrence of mode mixing, which is defined as a single Intrinsic Mode Function (IMF). IMFs can also consist of widely disparate scales, or can consist of a similar signal residing in different IMF components. Mode mixing is often a consequence of an intermittent signal, as discussed by Huang et al. [2, 3, 4]. An intermittent signal cannot only cause serious aliasing in the time-frequency distribution, but can also make the physical meaning of individual IMFs seriously unclear. To alleviate this drawback, Huang proposed the intermittence test $[3,2,4]$. This test aimed to avoid several difficulties. However the test caused its own issues, which were also illustrated by Huang and Wu. The first issue is the subjectively selected scale of the test. By this intervention EMD became totally adaptive. The second issue concerns separable and definable data selection from timescales, which was discussed by Huang and Wu et al. [4]. To overcome the scale separation issue without introducing a subjective intermittence test, Huang and Wu proposed a new Noise-Assigned Data Analysis (NADA) method, known as Ensemble EMD (EEMD), which defines the true IMF components as the mean value of an ensemble of trials [2]. Each trial consists of the signal plus a white noise of finite amplitude. Binding white noise helps to better cover real cases, as is common in current papers on image data analysis [1]. Adding white noise has shown EMD to be an adaptive dyadic filter bank. To make such an improvement, Wu and Huang were inspired by Flandring and Gledhill and their research in adding white noise to data analysis, which helps to improve EMD results [5, 4]. The main principle of EEMD was defined simply: "the added white noise would populate the whole time-frequency space uniformly with the constituting components of different scales. When a signal is added to this uniformly distributed white background, the bits of signal of different scales are automatically projected onto proper scales of reference established by the white noise in the background" [4, p. 2].

This approach was adopted on the basis of the following observations:

1. A collection of white noise cancels itself in the ensemble mean if averaged in a time domain; 
therefore, only the signal can survive and persist in the final noise-added signal ensemble when averaged.

2. Finite, not infinitesimal, amplitude white noise is needed to force the ensemble to exhaust all possible solutions. Finite magnitude noise makes the different scale signals reside in the corresponding IMF, dictated by the dyadic filter banks, and renders the resulting ensemble mean more meaningful.

3. The true and physically meaningful answer to EMD is not an answer without noise. It is designated to be the ensemble mean of a large number of trials consisting of the noise-added signal.

The proposed EEMD method utilizes many important statistical definitions of noise [4]. The following sections describe a part of the research done by Huang and $\mathrm{Wu}$ on the relation between white noise and a real signal. Image data is used in this paper. Section 2 provides a brief introduction to Ensemble Empirical Mode Decomposition, and several details of drawbacks associated with mode mixing are described. Section 3 describes the usefulness and capability of EEMD through examples in Image Data Analysis and Optical Flow Assignment.

\section{Introduction to Ensemble Empirical Mode Decomposition over EMD}

As an introduction to a more detailed description of the EEMD method, we begin with a short review of EMD $[2,6]$. The EMD method is an adaptive method, with the decomposition based on data and derived from data. In the EMD approach, the data, time series $x(t)$, is decomposed in terms of IMFs, as has been described in [4] by Huang,

$$
x(t)=\sum_{j=1}^{n} c_{n}+r_{n}
$$

where $r_{n}$ is the residue of the original data $x(t)$ and $n$ is the number of steps for extracting IMFs. IMFs are simple oscillatory functions with varying amplitudes and frequency. The extracted IMFs have the following properties:

1. Throughout the length of a single IMF, the number of extremes and the number of zero crossings must either be equal or must differ at most by one (although these numbers can differ significantly for the original data set).

2. At any data location, the mean value of the envelope defined by the local maxima and the envelope defined by the local minimum is zero.

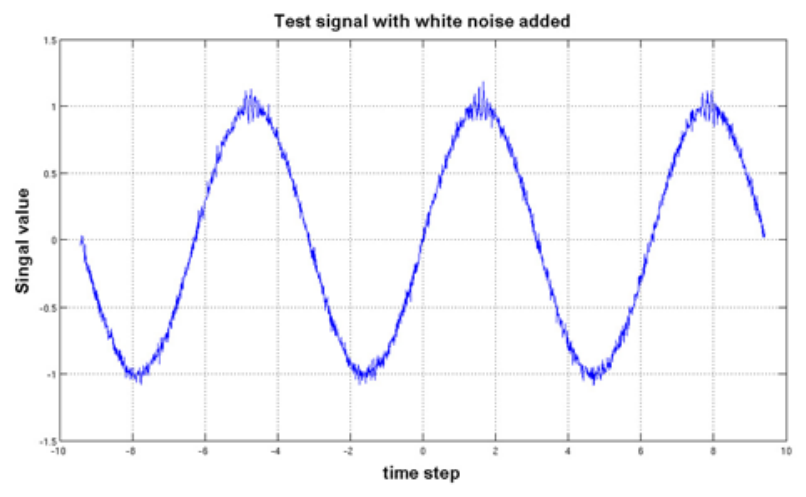

Fig. 1: The sine wave signal with three crests is used as an example in an introduction to EEMD (Fig. 2)
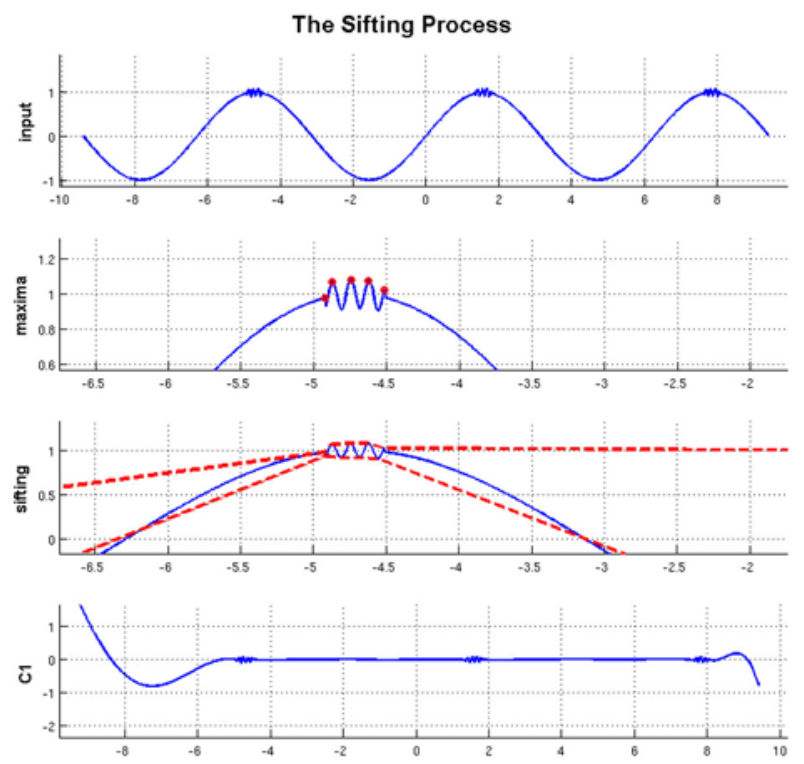

Fig. 2: The first step of the sifting process. Panel (a) is the input. Panel (b) identifies local highs (red dots). Panel (c) plots the upper envelope (upper red dashed line) and the lower envelope (lower red dashed line) and their input signal (blue line), and panel (d) are the difference between the input and the mean $m_{i}(t)$ of the envelopes

In common practice, EMD is implemented through a sifting process using only local extrema $[2$, $6]$. For any data $r_{j-1}$ the following procedure applies:

1. identify all the local extrema (a combination of highs and lows) and connect all these local highs (lows) with a cubic spline as the upper (lower) envelope

2. obtain the first component $h(t)$ by taking the mean $m(t)$ of the upper and lower envelopes

3. Treat $h(t)=x(t)-m(t)$ as the data, and repeat steps 1 and 2 as many times as is required until the envelopes are symmetric with respect to the zero mean under certain criteria.

The final $h(t)$ is designated as $c_{j}$. The complete sifting process stops when the residue $r_{n}$ becomes a monotone function from which no more IMFs can be 
extracted. The process is stopped using a stoppage criterion. Two types of stoppage criteria have commonly been used. The first type was used by Huang in 1998. It is based on a Cauchy type of convergence test. The test requires the normalized squared difference between two successive sifting operations defined as Eq. 1

$$
S D_{k}=\frac{\sum_{t=0}^{T}\left|h_{k-1}(t)-h_{k}(t)\right|^{2}}{\sum_{t=0}^{T} h_{k-1}^{2}}
$$

to be small. If this squared difference $S D_{k}$ is smaller than the desired number, the process will be stopped. Setting up the right $S D_{k}$ value seems to be a very difficult task, because no acceptable definition is available [2]. The second criterion is to set up a preselected $S$-number, which deals with other issues about how to select the appropriate number. These difficulties led authors [1] to develop a new approach to obtaining IMFs from the acquired signal (data), which will be described and applied below.

Based on the research of Fladrin and Gledhill, Wu and Huang [4] used their results as a background for improving the definition of the EMD method. Bearing the definition of EMD in mind, they showed that EMD behaves as a dyadic filter bank when white noise is populated uniformly through the whole timescale or time-frequency space [4].

Their description postulated that the total number of IMFs in a data set is close to $\log _{2}(N)$, with $N$ as the total number of data points. This fact ensures a total number of valid IMFs with difficulties around. When the signal is not merged with pure noise, some scales can be missing, and this involves the total number of IMFs, which can be lower than the expectation $\log _{2}(N)$. Another issue is caused by mode mixing, as in the previous EMD. An advantage of this approach is knowledge of the expected IMFs expressed in $N$ number.
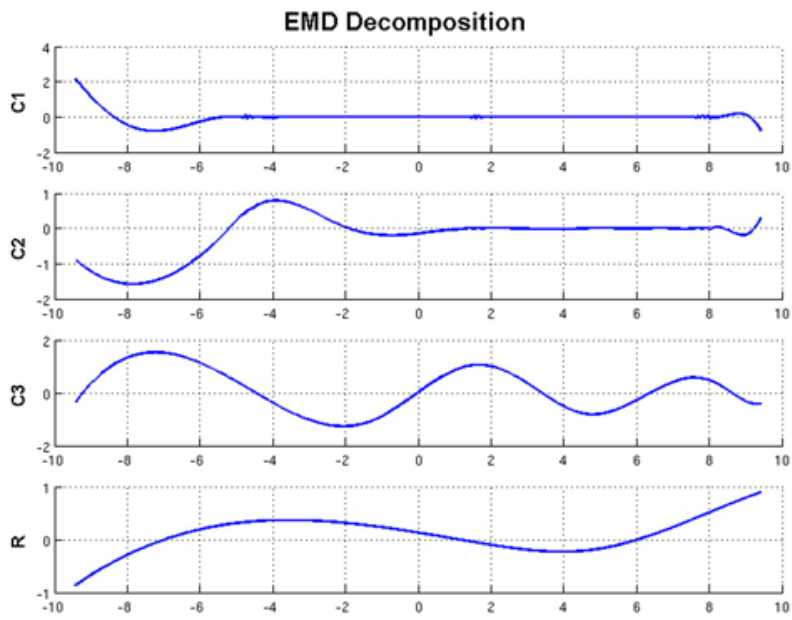

Fig. 3: The intrinsic mode functions of the input displayed in Fig. 1(a)

\subsection{A definition of mode mixing based on EMD}

Mode mixing has been defined as any IMF consisting of oscillation of dramatically disparate scales. When mode mixing occurs, IMF can cease to have a different physical meaning by itself. The signal can suggest an invalid physical representation. This known drawback was mentioned by Huang in $[2,3,4]$. An example of the sifting process is presented in Fig. 2, and its decomposition is shown in Fig. 3, as illustrated by Huang and $\mathrm{Wu}[4]$. The fundamental part of the data is the sine wave with unit amplitude. At the three middle crests of the sine wave, highfrequency intermittent oscillations with amplitudes close to 0.2 ride on the fundamental sine wave, in panel (a) of Fig. 2. This signal is denoted as the test signal. Panels (b) and (c) show the sifting process of the identifying highs (lows), as described above. Panel (d) displays the result, which is affected by visible mode mixing. This is because one envelope was a mixture of envelopes of the fundamental sine wave and the intermittent signal, leading to a severely distorted envelope mean, as shown in Fig. 2 and Fig. 4. Fig. 4 shows the main issue caused by appropriate localization of the highs and lows. An unpleasant implication of mode mixing leads to disadvantages of the original EMD method with the stoppage criterion, as described by Huang $[1,2,4]$. Mode mixing is also the main reason why the EMD algorithm is unstable (Fig. 3). Any small perturbation may result in a new set of IMFs, as reported by Gledhill [4]. Obviously, the intermittence prevents EMD from extracting any signal with similar scales. EEMD [4], which will be briefly described in the following sections, has been proposed to solve these problems.

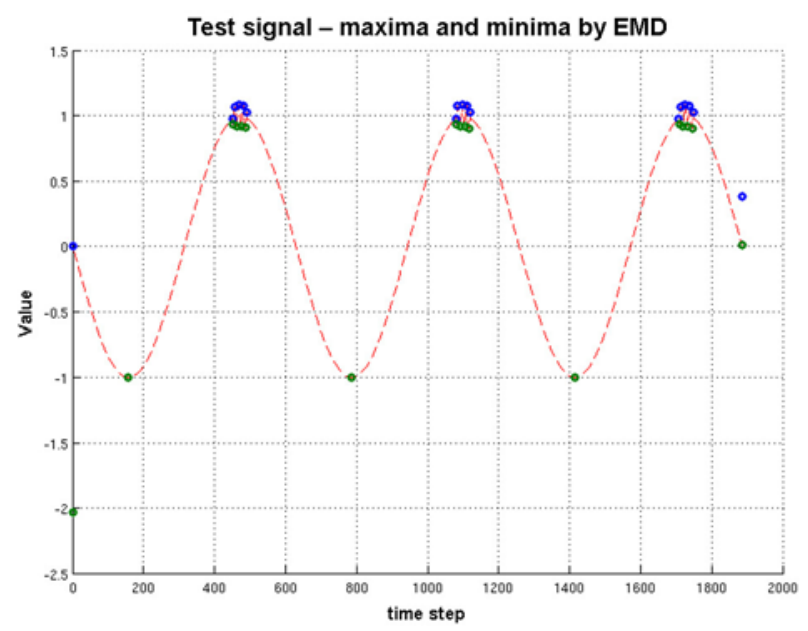

Fig. 4: Test signal (red dashed line) and the locations of highs (blue circle) and lows (green circle) 


\subsection{Ensemble empirical mode decomposition}

As shown in the previous test signal, the data (Fig. 2, Fig. 3) comprises collections of the main signal and some noise. To improve the precision of the measurements, the idea of the ensemble mean becomes a powerful approach. Data is collected by separate observations, each of which contains a different white noise realization [4].

To generalize this ensemble idea, the noise is populated to a single data set $x(t)$. The added white noise is treated as possible random noise that would be encountered in the measurement process. Under such conditions, the equation of any observation can be written as

$$
X_{t}(t)=x(t)+w_{i}(t)
$$

In this way, a different realization of white noise $w_{i}(t)$ will be added when there is only one observation. (Eq. 2)

We will now make a brief review of the properties of EMD before proceeding to a more detailed description of the EEMD method:

1. EMD is an adaptive data analysis method based on local characteristics of the data, and it therefore captures nonlinear, nonstationary oscillations more effectively

2. EMD is a dyadic filter bank for any Gaussian white noise-only series

3. when the data is intermittent, the dyadic property is often compromised in the original EMD, as shown in the example in Fig. 3

4. adding noise to the data can provide a uniformly distributed reference scale, which enables EMD to repair the compromised dyadic property

5. the corresponding IMFs of different series of white noise have no correlation with each other.

Therefore, the means $m_{i}(t)$ of the corresponding IMFs of different white noise series are likely to cancel each other.

Bearing in mind all these properties of the EMD method, the proposed EEMD has been developed in the following way:

1. add white noise series to the targeted data;

2. decompose the data with added white noise into the IMFs;

3 . repeat step 1 and 2 , based on the realization of different white noise series each time;

4. perform steps 1 and 2 until the IMFs of the data set are close to $\log _{2}(N)$, with $N$ as the number of total data points;

5. obtain the (ensemble) means of the corresponding IMFs of the decompositions as the final result.

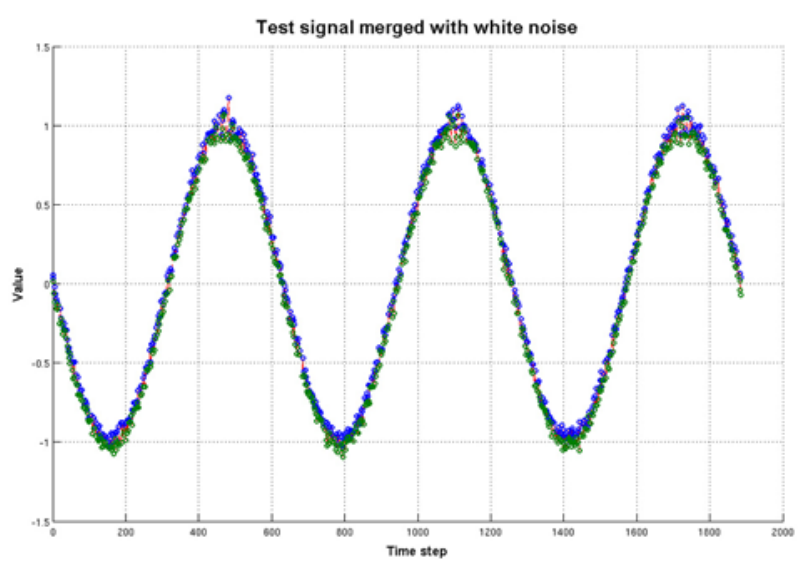

Fig. 5: Test signal with white noise added (red dashed line) and the location of its highs (blue circle) and lows (green circle)

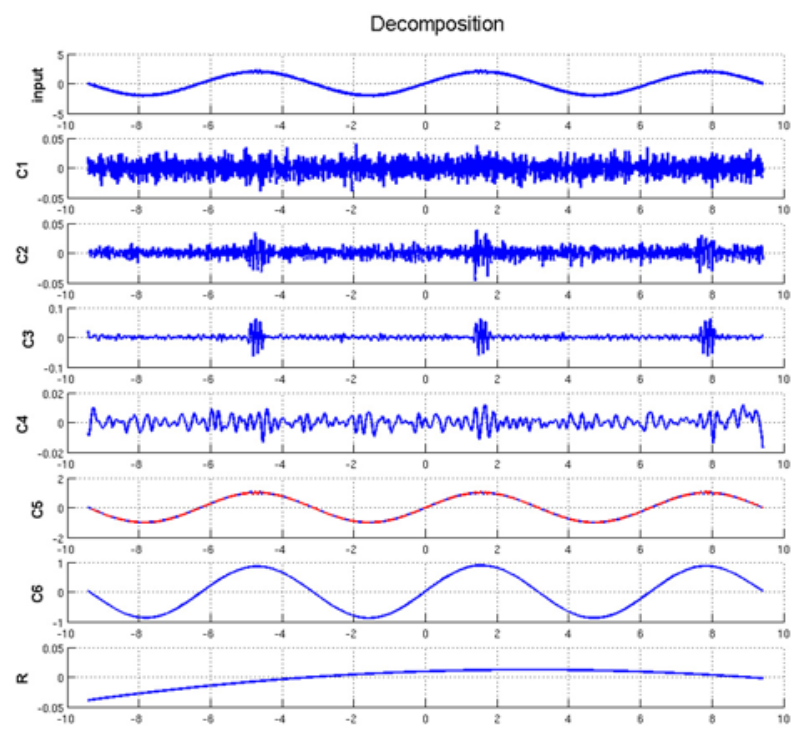

Fig. 6: The input top panel, its intrinsic mode functions (C1-6), and the trend (R). In panel C5, the original input is plotted as the bold dashed red line, for purposes of comparison

The main effect of decomposition using EEMD is that the added white noise series cancel each other in the final mean of the corresponding IMFs. This means that the IMFs stay within the natural dyadic filter windows, and thus significantly reduce the chance of mode mixing and preserve the dyadic property.

An example of the use of the EEMD method is illustrated in Fig. 6, in a similar meaning as for the previous EMD in Fig. 2 and Fig. 3. Clearly, the fundamental signal C5 is represented almost perfectly, as are the intermittent signals, if $\mathrm{C} 2$ and $\mathrm{C} 3$ are added together. The fact that the intermittent signal actually resides in two EEMD components is due to the average spectra of the neighboring IMFs of white noise overlapping, as revealed by $\mathrm{Wu}$ and Huang. It 
is necessary to combine the two adjacent components to one IMF. The need for this type of adjustment is easily determined through an orthogonal check. Whenever two IMF components become grossly nonorthogonal, we should consider combining the two components to form a single IMF component [4].

\section{EEMD method implemented in image data analysis}

The previous theoretical example shows the main concept of NADA, using the EEMD method as a tool. Based on previous text discussion, the question arises how the method could be used in image data analysis. Every frame of an image comprises a timesequence of data that is processed by the brain. Our brain gives an appropriate meaning to this ensemble of data. In this way, synapses can work as a dyadic filter bank. Data can be and mostly is affected by all kinds of noise. There can be various reasons for this noise. Examples are short-sightedness or daylight. The observed object is not sharply displayed on our retina and the data is treated in a damaged way. This can affect its meaning or its values. In this section, we will outline EEMD usage and how it can be used in analyzing such data. An example of cube rotation is displayed in Fig 7.

The brightness of the pixels over two images of the same cube in line height 120 pixels forms the test signal. The general size of the two images is $320 \times 240$ pixels in gray scale color mode. Gray scale mode was chosen for the sake of simplicity to better understand the mean value. In the first step, the two images were analyzed in a similar way. The line values were treated and the IMFs were extracted. As the deviation, the amount of white noise was set to 0.2 and the EEMD ensemble number was set to 50 .

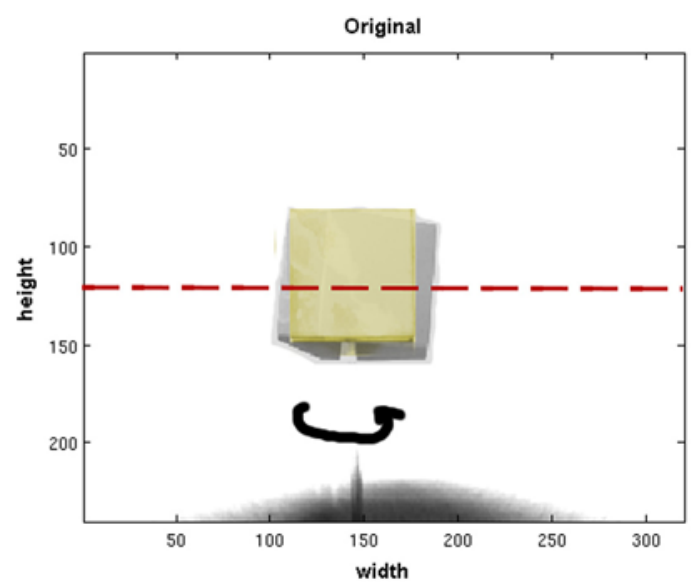

Fig. 7: Cube rotation: the cube rotates from a misaligned position to frontal position. The black arrow indicates the direction of rotation

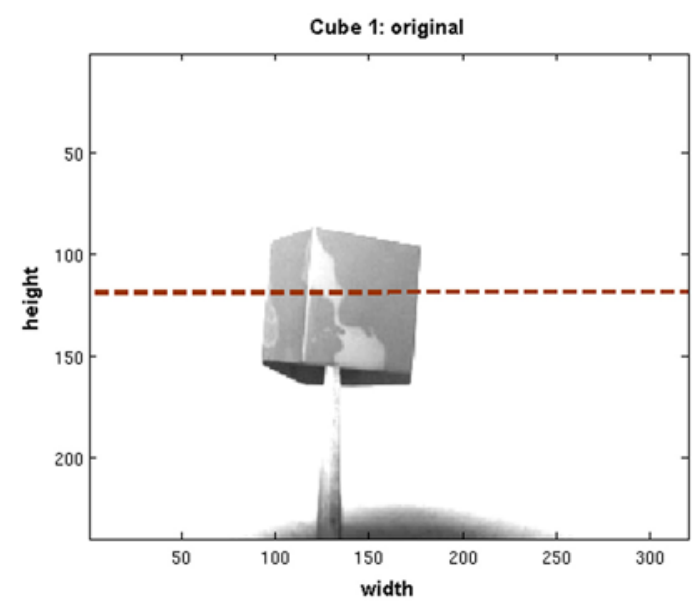

Fig. 8: Cube 1 - signal line

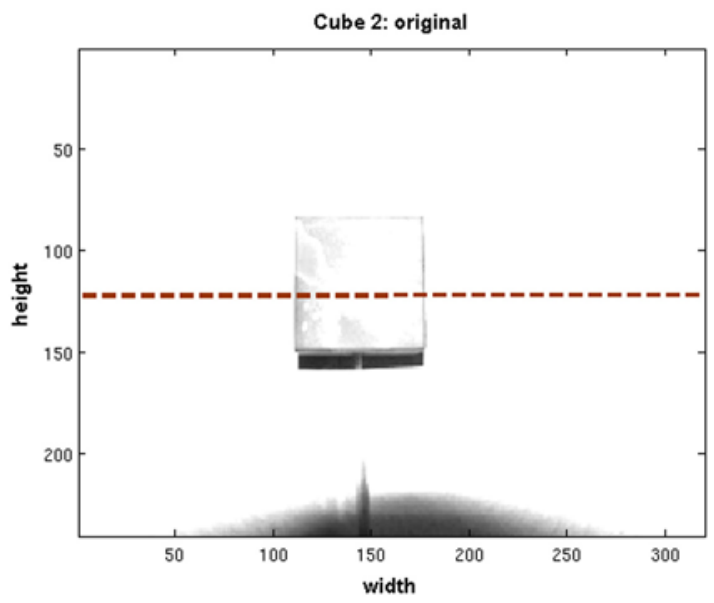

Fig. 9: Cube 2 - signal line

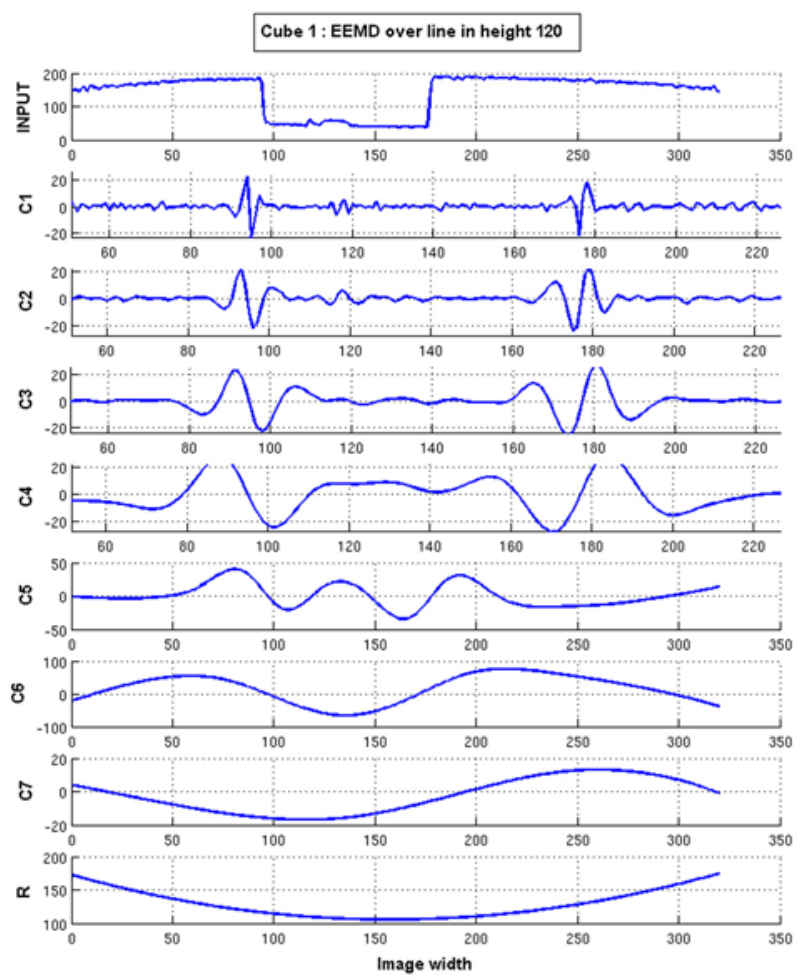

Fig. 10: Cube 1 - All IMF functions extracted by EEMD 
In the Cube 1 trial, the input (the original brightness data) is a visible jump around a width value of 100, which corresponds with the edge of the real cube (Fig. 7). Cube 1 is slightly dipped to the left side from the center, as shown in the original image (Fig. 8). The next peak in Fig. 10 of width of around 118 units can represent the second cube edge, which is coherent with reality. Consequent perturbation in the first IMF can reflect the footmark. The first main point here is the edge projection as a short perturbation with definable peaks over a zero value. Other IMFs can represent surface deviances. The last line is the residuum of the data. The residuum is mostly explained as a trend. The trend explanation can also be used for our case, because the last line shows a background change caused by the box. The original data defines the camber in the real scope. This jump is performed in the same way as in the trend line. Only eight different IMFs were extracted, which corresponds to the EEMD basics of the finite data set explained above. The data for Cube 2 (Fig. 9) is also treated and displayed in a similar sense as Cube 1 . The image of Cube 2 is taken from the front, which is also visible in the original. The data jump and the additional pit are smaller. This fact is caused by the projection of Cube 2, which is logical. The surface texture is also displayed in the original figure. As was described above, the EEMD method works with the IMF summary.

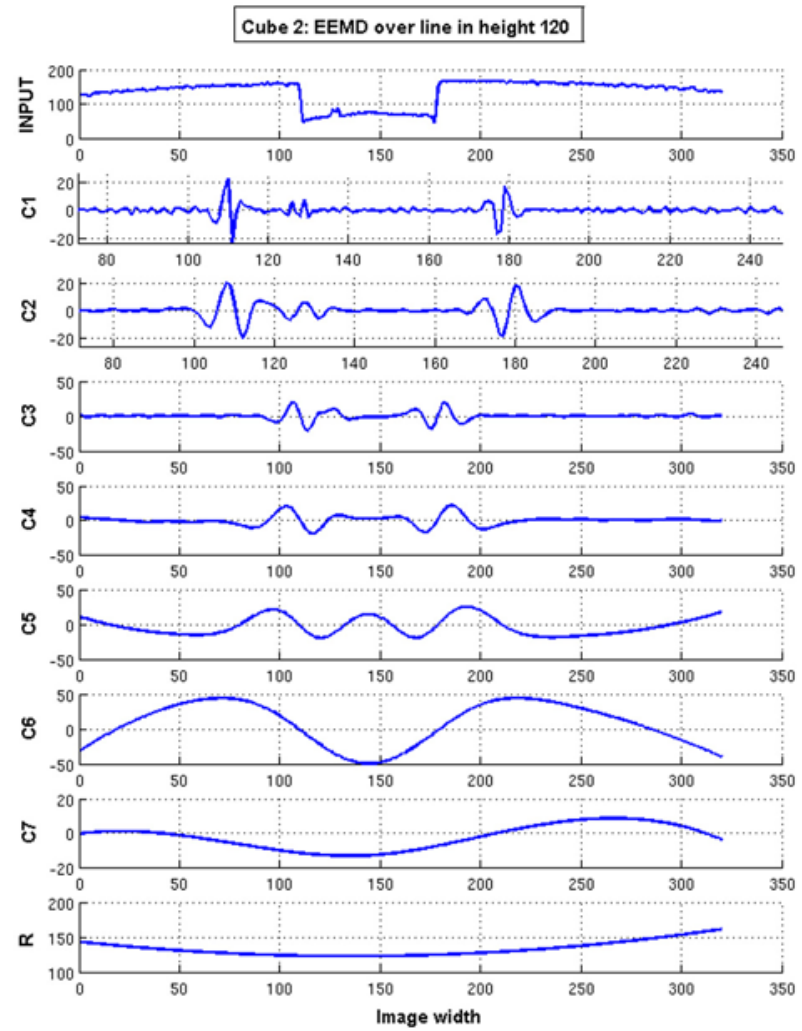

Fig. 11: Cube 2 - All IMF functions extracted by EEMD

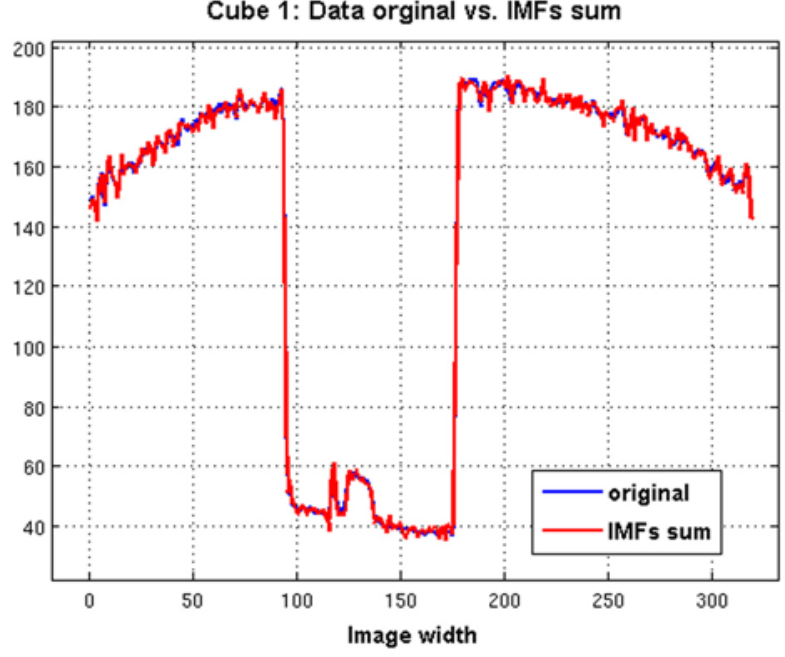

Fig. 12: Comparison between the original data set and the IMF summary

All IMFs should have no mutual correlation. IMFs should be independent of white-noise trials. This means that a summary of all IMFs should give us the original signal (Fig. 12). Finally, when we make a comparison of the two cubes, we find some kind of data shifts in the inputs and IMFs.

These shifts are treated by cube rotation from right to left, as indicated above (Fig. 7). There is no doubt that the two original figures are affected by different kinds of noise. The noise was treated during capture time. EEMD enables us to extract data from images properly, even in different layers. The main intention in edge detection is to find appropriate differences over all image layers, which is enabled by the use of the EEMD method presented here. Another important point is the logically different image width of cube rotation. Fig. 13 points out several areas where the cube edge can be projected on to the specific IMFs. Possible edge fluctuations are marked in subplot $\mathrm{C} 1$. These fluctuations are displayed as second fluctuations, because of the main transition. The first and the last fluctuations directly indicate a background change to/from the cube. Now we see that we have two limited areas where everything can be projected. In C2, the IMFs still show similar behavior over cubes with a logical width shift. The underlying change is shown in IMF C3. The Cube 1 line is projected with no underlying fluctuations, and it corresponds to reality with no additional cube edge. In Cube 2, however, the line fluctuation reaches a smaller peak and some kind of promontory is treated. This can be explained as a cube surface projection. When we look back to the original images, we see the true reality (Fig. 8 and Fig. 9). We consider here that C3 corresponds with the surface of the object where no inner edge fluctuation is displayed. This assertion is not proven, and 


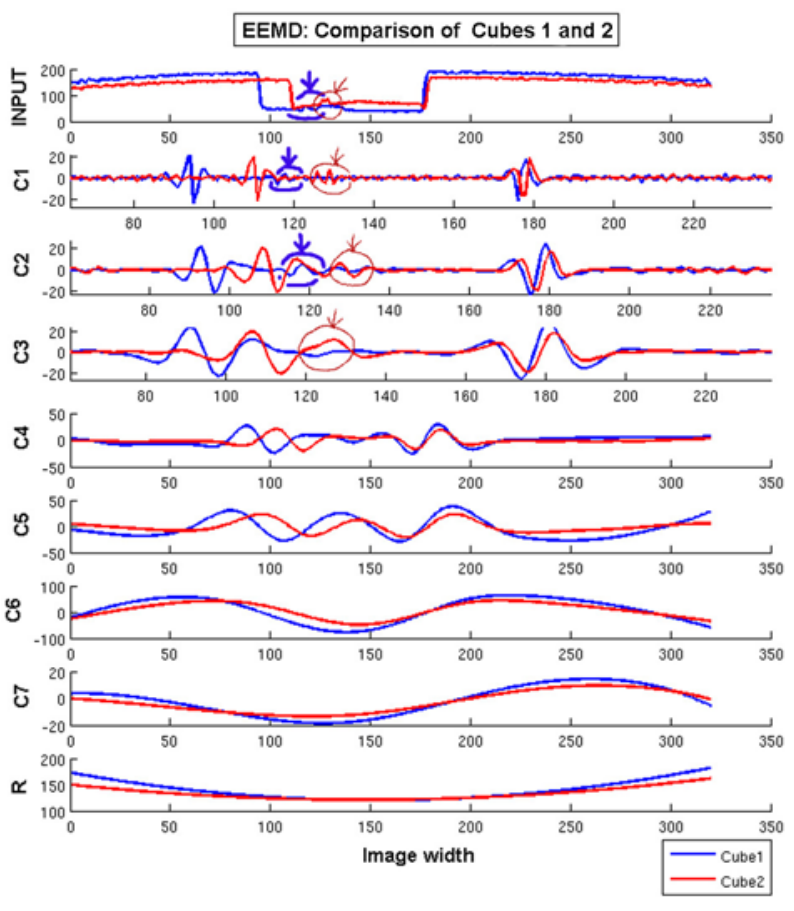

Fig. 13: Comparison of cube EEMDs - the colored circles indicate important edge detection areas

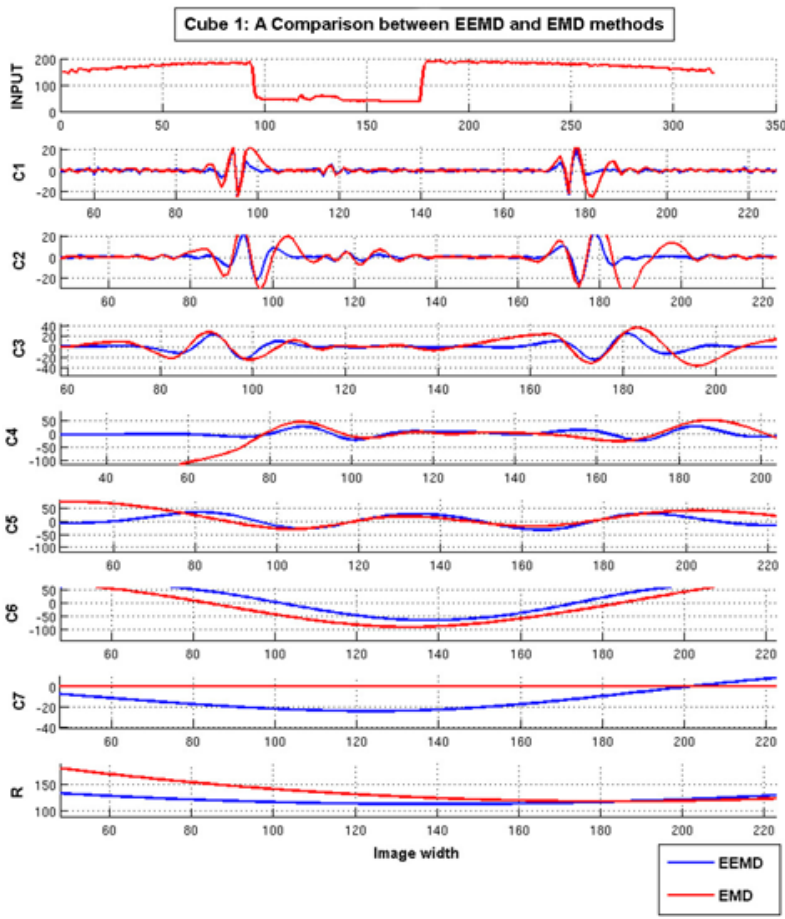

Fig. 14: Cube 1 - A comparison between EMD and EEMD methods and instability in analysis

will be a topic for further study. This result can be taken as a valid conclusion here. In our analysis of the Cube 1 signal, we found three edges. In the analysis of the Cube 2 signal we found only two edges, which correspond to the front view. The retrieved result is considered to be the cube rotation from the left dipped state to the right frontal view.

\section{A comparison between EMD methods and EEMD methods}

Fig. 14 presents a comparison between the original EMD method and the new EEMD method, using image data analysis.

\section{Conclusion}

A major drawback of EMD is the instability of the method, see Fig. 14. The EMD method has a problem even with edge detection. Edge fluctuations over the signal are more widely spread and are not as strict as in EEMD. EMD lines show a strong mixture of modes, where lines dramatically change direction with no valid reasons. Any edge projected on to the retina is a width limited area, which causes strict sensor stimulations. This assertion is closer to EEMD behavior, where narrower fluctuations are depicted.

In brief, results produced by the EMD method can be less valid than EEMD lines, which offer much stricter behaviors. This instability can have a dramatic effect on further studies of any signal, not necessary from an image $[1,2,4,6]$. The reason for the drawback is linked with the idea of a clear data signal. This idea does not correspond to real world examples. The EEMD method uses a white noise-added signal. This approach exhausts all possible solutions during the shifting process (Fig. 4, Fig. 5). The comparative test of the instability of the two methods displays the main benefit of using the EEMD method. The ability to divide the incoming signal data into more independent IMFs functions and then analyze it gives power to the EEMD method, where this signal, not necessary an image, can be interpreted and processed by layers. The natural fact of the independence of IMFs provides an opportunity to treat any signals as a summary of their layers, and this could be helpful in future medical research. There is potential and motivation for improving the proposed method.

\section{Acknowledgement}

This work was supported by the Grant Agency of the Czech Technical University in Prague, grant No. 2B06023 and by CTU grant SGS OHK2-021/10.

\section{References}

[1] Kopecký, M.: Hilbert-Huang Transformation and its application in optical flow evaluation, $S T C$, 2009.

[2] Huang, N. E., Samuel, S. P. Shen: Hilbert-Huang Transform and Its Application, Politecnico di $\mathrm{Mi}$ lano, Vol. 25. ISSN 0262-8856. 
[3] Huang, N. E., Shen, Z., Long, S. R., Wu, M. C., Shih, E. H., Zheng, Q., Tung, C. C., Liu, H. H.: The empirical mode decomposition method and the Hilbert spectrum for non-stationary time series analysis, Proc. Roy. Soc. London 454A, 1998, p. $903-995$.

[4] Wuand, Z., Huang, N. E.: Ensemble Empirical Mode Decomposition and Noise-assisted Data Analysis Method, World Scientific Publishing Copany, Advances in Adaptive Data Analysis, Vol. 1, No. 1 (2009), p. 1-41.

[5] Flandrin, P., Goncalves, P., Rilling, G.: EMD equivalent filter banks, from interpretation to applications, in Hilbert-Huang Transform: Intro- duction and Applications, eds. N. E. Huang and S. S. P. Shen, World Scientific, Singapore, 2005.

[6] Kokeš, J.: Okamžitá frekvence a HilbertHuangova transformace. Sborn $k$ konference Inteligentní systémy pro praxi, Lázně Bohdaneč, 2006. ISBN 80-239-6535-2.

Miroslav Kopecký

E-mail: Miroslav.Kopecky@fs.cvut.cz

Department of Instrumentation and Control Engineering

Czech Technical University in Prague

Faculty of Mechanical Engineering

Technická 2, 16627 Praha, Czech Republic 